Revue d'histoire de l'Amérique française

Q4. REVUE D'HISTOIRE DE L'AMÉRIQUE FRANÇAISE

\title{
Familles à prêtres? Familles à soeurs? Parenté et recrutement religieux au Saguenay (1882-1947)
}

\section{Gérard Bouchard}

Volume 48, numéro 4, printemps 1995

URI : https://id.erudit.org/iderudit/305362ar

DOI : https://doi.org/10.7202/305362ar

Aller au sommaire du numéro

Éditeur(s)

Institut d'histoire de l'Amérique française

ISSN

0035-2357 (imprimé)

1492-1383 (numérique)

Découvrir la revue

Citer cet article

Bouchard, G. (1995). Familles à prêtres? Familles à soeurs? Parenté et recrutement religieux au Saguenay (1882-1947). Revue d'histoire de l'Amérique française, 48(4), 483-508. https://doi.org/10.7202/305362ar
Résumé de l'article

Ce texte vise à faire ressortir l'influence de diverses variables familiales sur le recrutement du personnel religieux dans la région du Saguenay entre 1882 et 1947. Il établit d'abord que des facteurs comme la dimension et la structure de la famille, l'instabilité géographique et socioprofessionnelle, de même que le rang de naissance, étaient sans influence sur le recrutement. Par ailleurs, l'idée qu'un petit groupe de familles ait fourni un nombre proportionnellement très élevé de vocations (modèle des " pépinières familiales ") s'avère sans fondement. Cependant, il est vrai que l'Église avait tendance à recruter dans la parentèle (ou famille étendue). On observe aussi une certaine héritabilité (ou transmission familiale) de la propension à entrer en religion.

Enfin, l'étude conclut à l'importance des facteurs culturels pour expliquer le modèle de recrutement. Il reste toutefois à vérifier l'action de toutes les variables économiques.

Les données utilisées proviennent de répertoires de prêtres, de religieux et religieuses qui ont été jumelés au fichier BALSAC (pour la région du Saguenay). 


\title{
FAMILLES À PRÊTRES? FAMILLES À SCEURS? PARENTÉ ET RECRUTEMENT RELIGIEUX AU SAGUENAY (1882-1947) ${ }^{1}$
}

\author{
GÉRARD BOUCHARD \\ Institut interuniversitaire \\ de recherches sur les populations
}

(IREP)

\section{RÉSUMÉ}

Ce texte vise à faire ressortir l'influence de diverses variables familiales sur le recrutement du personnel religieux dans la région du Saguenay entre 1882 et 1947. Il établit d'abord que des facteurs comme la dimension et la structure de la famille, l'instabilité géographique et socioprofessionnelle, de même que le rang de naissance, étaient sans influence sur le recrutement. Par ailleurs, l'idée qu'un petit groupe de familles ait fourni un nombre proportionnellement très élevé de vocations (modèle des «pépinières familiales») s'avère sans fondement. Cependant, il est vrai que l'Église avait tendance à recruter dans la parentèle (ou famille étendue). On observe aussi une certaine héritabilité (ou transmission familiale) de la propension à entrer en religion.

Enfin, l'étude conclut à l'importance des facteurs culturels pour expliquer le modèle de recrutement. Il reste toutefois à vérifier l'action de toutes les variables économiques.

Les données utilisées proviennent de répertoires de prêtres, de religieux et religieuses qui ont été jumelés au fichier BALSAC (pour la région du Saguenay).

\section{ABSTRACT}

The aim of this paper is to bring out the influence of several family variables on the recruitment of the Catholic Church (priests and members of religious communities) in the Saguenay region between 1882 and 1947. First, the study establishes that such factors as family size and structure, geographic and occupational mobility as

1. Les travaux qui ont conduit à cet article ont pu être réalisés grâce à des subventions du Conseil de recherches en sciences humaines du Canada, du Fonds FCAR (Québec) et de l'Université du Québec à Chicoutimi. Par ailleurs, l'auteur tient à remercier tout particulièrement Régis Thibeault, $D^{r}$ Marcel Thivierge, Michèle Jomphe, Jocelyn Côté, Martine Dufour et Raymond Roy (production et compilation des données), Pierre Jacques (collaboration à la constitution du corpus du personnel religieux), Judith Goulet et Carole Roy (traitement de texte), Pascale Dupont et Robin Simard (traitement informatique des données), Guy Laperrière et Marc Saint-Hilaire (relecture et critique d'une version précédente). 
well as birth order had no impact upon recruitment. Also, the idea that a few families provided a very large share of Church officials proves groundless. On the other hand, there was a tendency for the Church to recruit among the wider family, and the data show some heritability (or family transmission) in the propensity to "answer the call».

Finally, the study underlines the importance of cultural factors in accounting for the recruitment pattern. This statement, however, may need qualifying since the analysis of economic factors - such as family wealth - still has to be carried out.

The study combines information from lists of priests and members of religious orders and from the BALSAC data base (for the Saguenay region).

Ce texte est le dernier d'une série de trois articles consacrés à l'histoire sociale du personnel religieux dans la région du Saguenay (englobant la sous-région du Lac-Saint-Jean). Le premier, qui portait sur les communautés de religieuses ${ }^{2}$, traitait de l'évolution numérique ainsi que de la provenance géographique et sociale des membres. Parmi les principaux résultats, l'enquête établissait que les religieuses représentaient près des trois quarts du personnel religieux (incluant les prêtres, les pères et les frères) et qu'elles étaient, selon les souspériodes, de quatre à six fois plus nombreuses que les prêtres; on apprenait aussi que leur recrutement commençait à décroître dès la décennie 1932-19413. En outre, il n'a pas été possible de déceler dans ce recrutement des éléments de différenciation spatiale ou sociale démontrant un véritable modèle préférentiel, les écarts en termes socioprofessionnels s'expliquant surtout par des proximités géographiques par rapport aux maisons-mères, couvents et autres institutions. Dans un deuxième texte ${ }^{4}$, l'enquête a été étendue à toutes les catégories du personnel religieux. Cette fois, il est apparu que, sur le plan spatial, le recrutement des prêtres privilégiait légèrement le milieu urbain. De plus, en ce qui concerne les origines sociales, une stratification se manifestait chez l'ensemble des hommes, et plus particulièrement chez les prêtres. Ici, il s'avère que la contribution de la

2. Gérard Bouchard, Raymond Roy, Pierre Jacques, «La composition des communautés de religieuses au Saguenay (1882-1947)», La Société canadienne d'histoire de l'Église catholique, Sessions d'étude, 55 (1988): 87-117.

3. D'après N. Perron qui a étudié la communauté des Augustines Hospitalières, le déclin se serait probablement manifesté plus tôt, n'eût été certaines réformes dans les conditions de vie. Normand Perron, Un siècle de vie hospitalière au Québec. Les Augustines et l'Hôtel-Dieu de Chicoutimi, 1884-1984 (Sillery et Chicoutimi, Presses de l'Université du Québec et Les Augustines de la Miséricorde de Jésus, 1984), 242 et ss. Les résultats présentés à l'échelle du Québec par N. Laurin et al. recoupent les nôtres sur ce point. N. Laurin, D. Juteau, L. Duchesne, À la recherche d'un monde oublié. Les communautés religieuses de femmes au Québec de 1900 à 1970 (Montréal, Le Jour éditeur, 1991), 424 p.

4. G. Bouchard et R. Thibeault, «Origines géographiques et sociales du personnel religieux dans la région du Saguenay». À paraître dans Histoire sociale/Social History. 
petite bourgeoisie excédait largement la part de ses effectifs dans la population régionale. Par ailleurs, il n'a pas été possible d'identifier des pratiques sélectives de recrutement en faveur de la paysannerie, contrairement à ce qu'annonçait une idée très répandue dans l'historiographie québécoise.

Dans le présent essai, nous nous interrogeons sur les paramètres strictement familiaux du recrutement religieux. La première partie sera consacrée à des variables comme l'alphabétisation, la dimension et la structure de la famille, le rang de naissance, etc. Dans une deuxième partie, nous étudierons différents aspects de l'apparentement en rapport avec l'entrée en religion.

Tout comme les précédentes, ces analyses ont été conduites à l'échelle du Saguenay àl'aide, d'abord, d'un répertoire de l'ensemble des prêtres, religieux, religieuses et frères natifs de cette région ayant été ordonnés ou ayant prononcé leurs vœux entre 1882 et 1947 . Ce répertoire a été établi par $\mathrm{M}^{\mathrm{gr}} \mathrm{F}$.-X.-E. Frenette ${ }^{5}$; il contient le signalement de tous les clercs, religieux et religieuses originaires du diocèse de Chicoutimi, ainsi que des clercs y ayant travaillé. Le document donne a) les dates de naissance, d'entrée en religion ou d'ordination et, éventuellement, de décès; b) le nom de la communauté, s'il y a lieu; c) l'identité et, souvent, la profession des parents. Ces données, qui ont fait l'objet de plusieurs vérifications critiques ${ }^{6}$, ont été informatisées (fichier sectoriel RELIG) et intégrées par jumelage au fichier de population de l'IREP (BALSAC) ${ }^{7}$. Ce dernier fichier constitue la deuxième source utilisée. À l'aide du système de gestion de base de données INGRES/INGRID, les dossiers des membres du personnel religieux ont ainsi été jumelés à leur fiche de famille, ce qui a donné accès à toutes les variables microsociales qui seront traitées ci-après ${ }^{8}$.

5. F.-X.-Eugène Frenette (Chanoine), Notices biographiques et notes historiques sur le diocèse de Chicoutimi (Chicoutimi, Chancellerie de l'Évêché, 1945), 243 p.; Supplément aux "Notices biographiques et notes historiques» sur le diocèse de Chicoutimi (Chicoutimi, Chancellerie de l'Évêché, 1947), 415 p.

6. Notamment à l'aide d'un deuxième répertoire contenant la liste des prêtres séculiers ayant œuvré dans le diocèse jusqu'en 1968. André Simard, Les évêques et les prêtres séculiers au diocèse de Chicoutimi, 1878-1968 (Chicoutimi, Chancellerie de l'Évêché, 1969), 812 p.

7. On notera que d'un tableau à l'autre et en fonction des variables étudiées, le nombre total d'individus varie à cause de certaines lacunes dans les données (ex.: calcul des rangs de naissance).

8. Le fichier de population BALSAC contient les 815000 actes de l'état civil du Saguenay entre 1842 et 1986 . Il permet l'accès aux 125000 familles reconstituées, à leur généalogie et à divers fichiers dits sectoriels analogues à celui des clercs et religieux. Sur le contenu, la structure et le fonctionnement du fichier-réseau BALSAC, voir G. Bouchard, R. Roy et B. Casgrain, Reconstitution automatique des familles. Le système SOREP (Université du Québec à Chicoutimi, 1985), 2: 745 p.; «De la micro à la macro-reconstitution des familles. Le système SOREP», GENUS, 42,3-4 (décembre 1986): 33-54, de même que les Documents I-C42, I-C-53 et I-C-76 de l'IREP. 
Le fichier de population a également servi à construire ce qu'on pourrait appeler la population de base, au sein de laquelle a été prélevée la sous-population du personnel religieux. Cette population de base consiste, pour diverses sous-périodes, dans l'ensemble des jeunes gens natifs du Saguenay qui ont survécu jusqu'à l'âge du mariage; il s'agit bel et bien de la population susceptible d'entrer en religion. Nous parlerons de population de référence pour la désigner; elle servira de terme de comparaison tout au long de cette enquête.

Un mot enfin sur la terminologie. Nous recourrons aux expressions gens d'Église et personnel religieux pour désigner l'ensemble des prêtres, pères, frères (enseignants, convers) et religieuses. Aussi, à l'exemple de B. Denault et $\mathrm{B}$. Lévesque ${ }^{9}$, le concept de communauté sera utilisé dans son sens générique, incluant les ordres, les congrégations, les sociétés, instituts et états religieux ${ }^{10}$.

\section{1 - LE MILIEU FAMILIAL DU PERSONNEL RELIGIEUX}

Nous avons testé diverses variables pour caractériser - le cas échéant - le type d'environnement familial susceptible de favoriser ce qu'on appelait couramment les vocations religieuses. Les dimensions explorées vont de la culture à la démographie; elles recouvrent tout l'éventail des données qui nous étaient disponibles.

\section{A - L'alphabétisation}

De nombreux auteurs" ${ }^{11}$ ont constaté ou fait l'hypothèse d'une corrélation négative entre la scolarisation et le recrutement religieux, nourrissant ainsi l'idée que l'Église québécoise aurait misé surtout sur la foi des humbles pour former ses rangs - plutôt que sur la contribution des milieux les plus «éclairés». Le test auquel nous avons pu procéder ne repose pas sur une mesure directe de la scolarité mais sur un indice d'alphabétisation des pères de famille ayant contribué au recrutement religieux. Il s'agit, en l'occurrence, d'un nouvel indice

9. Bernard Denault et Benoît Lévesque, Éléments pour une sociologie des communautés religieuses au Québec (Montréal et Sherbrooke, Presses de l'Université de Montréal et Université de Sherbrooke, 1975), 220 p.

10. Voir aussi à ce propos: G. Bouchard, R. Roy et P. Jacques, loc. cit., 90-91; Raoul Naz, Dictionnaire du droit canonique: contenant tous les termes du droit canonique... (Paris, Letouzey et Ane, 1935-1965), 7 vol.; Jean Hamelin et Nicole Gagnon, Histoire du catholicisme québécois: le XXe siècle, tome 1: 1898-1940 (Montréal, Boréal Express, 1984), 144-145.

11. Dont Serge Gagnon, Louise Lebel-Gagnon, «Le milieu d'origine du clergé québécois, 1775-1840: mythes et réalités», Revue d'histoire de l'Amérique française, 37,3 ( décembre 1983): 373-397. 
développé à l'IREP ${ }^{12}$. Fondé sur l'exploitation des fiches de familles reconstituées, l'indice PMP est calculé en tenant compte de tous les actes (baptême, mariage, sépulture) qui, dans une fiche de famille, donnent une information sur l'aptitude à signer son nom - alors que l'indice classique ne retient que la signature des conjoints dans l'acte de mariage ${ }^{13}$. Par rapport à ce dernier, l'indice PMP présente plusieurs avantages, dont celui de refléter les niveaux et les degrés d'alphabétisation. Ses valeurs, exprimées en pourcentage, varient entre 0 et 100 . On se rappellera que l'indice classique ne fournit que des valeurs binaires: alphabétisé(e)/analphabète. Nous avons pu montrer aussi, à l'aide des manuscrits des recensements du XIX ${ }^{\mathrm{e}}$ siècle, qu'à certains seuils de l'indice correspondaient des aptitudes spécifiques, comme savoir lire ou savoir écrire. L'indice PMP ne peut toutefois être calculé que pour les pères, étant donné que les actes de baptême et de sépulture reproduisent très partiellement l'information relative à l'aptitude des femmes à signer ${ }^{14}$.

Le PMP moyen a été calculé pour les pères du personnel religieux et pour les pères des témoins (population de référence) (tableau 1). D'autres calculs ont été effectués avec des variantes de l'indice PMP (ex.: \% des pères ayant un PMP $>80 \%$ ); ils donnent tous des résultats convergents. Quelle que soit la sous-période ou la décennie considérée, les parents du groupe cible sont toujours nettement plus alphabétisés que ceux du groupe témoin ${ }^{15}$. Dans l'ensemble de la période, l'écart moyen est de 10 points en faveur du premier. Comme on s'y attendait, les familles des prêtres (jusqu'au début du $\mathrm{XX}^{\mathrm{e}}$ siècle), et en particulier celles des pères, montrent de hauts niveaux d'alphabétisation. Il en va de même pour les frères, ce qui surprend un peu compte tenu de la place très modeste qui leur était souvent attribuée dans la hiérarchie officieuse des statuts religieux.

À la lumière de ces résultats, il serait exagéré de conclure que l’Église saguenayenne recrutait ses membres parmi l'élite culturelle;

12. G. Bouchard et J. Larouche, «Nouvelle mesure de l'alphabétisation à l'aide de la reconstitution automatique des familles», Histoire sociale/Social History, 22,43 (mai 1989): 91119; G. Bouchard, «Évolution de l'alphabétisation (masculine) au Saguenay: les variables géographiques, 1842-1971», Historical Papers/Communications historiques, (1989): 13-35; «Computerized Family Reconstitution and the Measure of Literacy. Presentation of a New Index», History and Computing, 5,1 (1993): 13-24.

13. PMP pour: proportion de mentions positives (portant la signature de l'individu) dans une fic.he de famille donnée.

14 Ainsi, parce que la cérémonie suivait de trop près la naissance de l'enfant, la mère n'assistait presque jamais à son baptême. L'historien doit composer avec cette contrainte.

15. Comme nous ne traitons pas ici avec des échantillons mais avec des populations totales, nous n'avons pas cru utile de recourir à des tests statistiques destinés à établir le caractère significatif des écarts observés. 
Tableau 1

Alphabétisation comparée des pères du personnel religieux et des témoins

Saguenay, 1882-1947

\begin{tabular}{|c|c|c|c|c|c|c|c|c|c|c|c|c|c|c|}
\hline \multirow[t]{2}{*}{$\begin{array}{l}\text { Sous- } \\
\text { périodes }\end{array}$} & \multicolumn{2}{|c|}{$\begin{array}{c}\text { Prêtres } \\
\text { (1) }\end{array}$} & \multicolumn{2}{|c|}{$\begin{array}{l}\text { Pères } \\
\text { (2) }\end{array}$} & \multicolumn{2}{|c|}{$\begin{array}{c}\text { Frères (c) } \\
\text { (3) }\end{array}$} & \multicolumn{2}{|c|}{$\begin{array}{l}\text { Hommes } \\
\text { (religieux) } \\
(1+2+3)\end{array}$} & \multicolumn{2}{|c|}{$\begin{array}{l}\text { Sœurs } \\
\text { (4) }\end{array}$} & \multicolumn{2}{|c|}{$\begin{array}{c}\text { Personnel } \\
\text { religieux } \\
(\text { total } 1+2+3+4)\end{array}$} & \multicolumn{2}{|c|}{ Têmoins } \\
\hline & $\begin{array}{c}\text { N. de } \\
\text { familles }\end{array}$ & $\begin{array}{c}\text { PMP } \\
\text { moyen }\end{array}$ & $\begin{array}{c}\text { N. de } \\
\text { familles }\end{array}$ & $\begin{array}{c}\text { PMP } \\
\text { moyen }\end{array}$ & $\begin{array}{c}\text { N. de } \\
\text { familles }\end{array}$ & $\begin{array}{c}\overline{P M} \bar{P} \\
\text { moyen }\end{array}$ & $\begin{array}{c}\text { N. de } \\
\text { familles }\end{array}$ & $\begin{array}{l}\text { PMP. } \\
\text { moyen }\end{array}$ & $\begin{array}{l}\text { N. de } \\
\text { familles }\end{array}$ & $\begin{array}{l}\text { PMP } \\
\text { moyen }\end{array}$ & $\begin{array}{c}\text { N. de } \\
\text { familles }\end{array}$ & $\begin{array}{c}\text { PMP } \\
\text { moyen }\end{array}$ & $\begin{array}{c}\text { N. de } \\
\text { familles }\end{array}$ & $\begin{array}{l}\text { PMP } \\
\text { moyen }\end{array}$ \\
\hline $1882-1891$ & 11 & 23,6 & - & - & 3 & 11,1 & 14 & 20,1 & 30 & 13,2 & 44 & 15,4 & 1569 & 10,7 \\
\hline $1892-1901$ & 19 & 29,4 & - & - & 4 & 30,9 & 23 & 29,7 & 56 & 25,5 & 79 & 26,8 & 1825 & 17,2 \\
\hline $1902-1911$ & 19 & 43,5 & 1 & 72,2 & 5 & 36,7 & 25 & 43,3 & 83 & 40,6 & 108 & 41,2 & 2259 & 25,8 \\
\hline $1912-1921$ & 24 & 52,3 & 6 & 53,8 & 17 & 56,3 & 47 & 54,0 & 129 & 50,3 & 176 & 51,3 & 3144 & 39,8 \\
\hline $1922-1931$ & 52 & 59,9 & 11 & 78,4 & 24 & 76,4 & 87 & 65,9 & 240 & 66,6 & 327 & 66,4 & 3820 & 55,2 \\
\hline $1932-1941$ & 51 & 73,4 & 34 & 70,9 & 52 & 82,2 & 137 & 76,3 & 266 & 75,8 & 403 & 76,0 & 5839 & 69,3 \\
\hline $1942-1947$ & 22 & 77,2 & 13 & 88,9 & 36 & 86,7 & 71 & 84,6 & 101 & 78,1 & 172 & 80,6 & 5406 & 79,1 \\
\hline $1882-1947$ & 198 & 57,7 & 65 & 74,6 & 141 & 74,1 & 404 & 66,1 & 905 & 62,2 & 1309 & 63,3 & 23862 & 53,5 \\
\hline
\end{tabular}

Notes : a) Seules les familles ayant fourni un seul enfant à l'Église ont été retenues.

b) Un enfant a êté tiré au hasard dans chaque famille-témoin.

Sources:

Fichier sectoriel RELIG

Frères enseignants et frères convers

Fichier BALSAC (Saguenay)

d) $\quad \mathrm{N}=$ Nombre absolu 
par contre, elle ne privilégiait certainement pas les milieux sous-alphabétisés, loin de là. Cette conclusion s'explique en partie du fait que le groupe cible ne reproduit pas exactement la structure socioprofessionnelle de la population de référence; on se rappellera en effet que, proportionnellement, un plus grand nombre de recrues provenaient de la petite bourgeoisie. Néanmoins, l'écart observé sur l'alphabétisation se marque aussi chez les religieuses qui, elles, étaient recrutées de façon beaucoup plus également dans les différents milieux sociaux. C'est bien le signe d'une certaine sélection en faveur de familles un peu plus scolarisées que la moyenne.

\section{$B$ - La dimension de la famille}

Dans ce deuxième test, seule la taille de la famille est prise en considération. La question posée se formule ainsi: proportionnellement, le nombre des vocations était-il plus élevé dans les familles nombreuses? Cette précision est importante car, lorsqu'il s'agit d'évaluer l'influence de la famille, on confond souvent quatre phénomènes bien différents: l'effet de la dimension du groupe familial, le bassin familial de recrutement (dans l'ensemble des familles? ou dans un groupe restreint, sorte de «pépinières» familiales?), la contribution de la parentèle (famille «étendue»), et l'effet intergénérationnel ou héritabilité. En ce qui concerne le premier phénomène, pratiquement tous les auteurs prennent pour acquis que les membres de l'Église québécoise provenaient davantage de familles nombreuses. Il y a déjà longtemps, à l'instar de Lionel Audet ${ }^{16}$, Albert Tessier ${ }^{17}$ l'affirmait au terme d'une étude sur un groupe de religieuses. De leur côté, Jean Hamelin et Nicole Gagnon ${ }^{18}$ rappellent que «partout dans le monde, les familles nombreuses sont proportionnellement plus fécondes en vocations». La même conclusion ressort des travaux de Nicole Laurin, Danielle Juteau et Lorraine Duchesne ${ }^{19}$ sur les religieuses québécoises entre 1900 et $1970^{20}$.

16. Lionel Audet, «Familles nombreuses: pépinières de vocations», La Semaine religieuse de Québec (29 juin 1950): 696-698.

17. Albert Tessier, «Onze enfants, mais c'est immoral!», Relations, 35 (novembre 1943): 296-297.

18. J. Hamelin et N. Gagnon, Histoire du catholicisme québécois, op. cit., 154.

19. N. Laurin, D. Juteau et L. Duchesne, op. cit., 302-317.

20. Sur le même sujet et dans le même sens, voir aussi Marc-A. Lessard, Jean-Paul Montminy, «Recensement des religieuses au Canada», Donum Dei, 11, Conférence religieuse canadienne (Ottawa, 1966), 389 p. Paul Stryckman, Les prêtres au Québec aujourd'hui. Résultats d'une recherche sociologique (Québec, Centre de recherche en sociologie religieuse, Université Laval, 1970), 473 p., 2 vol. Également, une série de textes publiés par le père A. Fortier dans l'Action catholique en 1953 (9 avril, 26 et 28 mai, 14 septembre). 
Tableau 2

Dimensions comparées (nombre moyen de naissances vivantes) des familles

d'origine des prêtres et des témoins, selon le groupe professionnel

Saguenay, 1842-1947

\begin{tabular}{|c|c|c|c|c|c|c|c|c|c|c|c|c|}
\hline \multirow{3}{*}{$\begin{array}{c}\text { Sous- } \\
\text { périodes }\end{array}$} & \multicolumn{4}{|c|}{ Cultivateurs } & \multicolumn{4}{|c|}{ Autres professions } & \multicolumn{4}{|c|}{ Toutes professions } \\
\hline & \multicolumn{2}{|c|}{ Prêtres } & \multicolumn{2}{|c|}{ Témoins } & \multicolumn{2}{|c|}{ Prêtres } & \multicolumn{2}{|c|}{ Témoins } & \multicolumn{2}{|c|}{ Prêtres } & \multicolumn{2}{|c|}{ Témoins } \\
\hline & $\begin{array}{c}N . \text { de } \\
\text { familles }\end{array}$ & $\begin{array}{l}\text { N. moyen } \\
\text { d'enfants }\end{array}$ & $\begin{array}{c}\text { N. de } \\
\text { familles }\end{array}$ & $\begin{array}{l}\text { N. moyen } \\
\text { d'enfants }\end{array}$ & $\begin{array}{c}\text { N. de } \\
\text { familles }\end{array}$ & $\begin{array}{l}\text { N. moyen } \\
\text { d'enfants }\end{array}$ & $\begin{array}{c}\text { N. de } \\
\text { familles }\end{array}$ & $\begin{array}{l}\text { N. moyen } \\
\text { d'enfants }\end{array}$ & $\begin{array}{c}\text { N. de } \\
\text { familles }\end{array}$ & $\begin{array}{l}\text { N. moyen } \\
\text { d'enfants }\end{array}$ & $\begin{array}{l}\text { N. de } \\
\text { familles }\end{array}$ & $\begin{array}{l}\text { N. moyen } \\
\text { d'enfants }\end{array}$ \\
\hline $1882-1899$ & 7 & 12,6 & 609 & 11,1 & 6 & 10,0 & 143 & 10,0 & 13 & 11,3 & 752 & 10,6 \\
\hline $1900-1947$ & 70 & 11,1 & 3506 & 10,9 & 52 & 9,8 & 2439 & 10,0 & 122 & 10,5 & 5945 & 10,5 \\
\hline Ensemble & 77 & 11,2 & 4115 & 11,0 & 58 & 9,8 & 2582 & 10,0 & 135 & 10,6 & 6697 & 10,5 \\
\hline
\end{tabular}

Sources: $\quad$ Fichier sectoriel RELIG

Fichier BALSAC (Saguenay) 
Tableau 3

Dimensions comparées (nombre de naissances vivantes) des familles d'origine des pères, des frères et des témoins, selon le groupe professionnel Saguenay, 1900-1947 (a)

\begin{tabular}{||c|c|c|c|c|c|c||}
\hline \multirow{2}{*}{$\begin{array}{c}\text { Groupes } \\
\text { professionnels }\end{array}$} & \multicolumn{2}{|c|}{ Cultivateurs } & \multicolumn{2}{c|}{ Autres professions } & \multicolumn{2}{c|}{ Toutes professions } \\
\cline { 2 - 7 } & $\begin{array}{c}\text { N. de } \\
\text { familles }\end{array}$ & $\begin{array}{c}\text { N. moyen } \\
\text { d'enfants }\end{array}$ & $\begin{array}{c}\text { N. de } \\
\text { familles }\end{array}$ & $\begin{array}{c}\text { N. moyen } \\
\text { d'enfants }\end{array}$ & $\begin{array}{c}\text { N. de } \\
\text { familles }\end{array}$ & $\begin{array}{c}\text { N. moyen } \\
\text { d'enfants }\end{array}$ \\
\hline Pères & 19 & 11,3 & 25 & 9,4 & 44 & 10,2 \\
Frères & 46 & 11,5 & 39 & 11,0 & 85 & 11,3 \\
\hline Témoins & 3506 & 10,9 & 2439 & 10,0 & 5945 & 10,5 \\
\hline
\end{tabular}

(a) La période 1882-1899 n'apparaît pas à cause des petits effectifs.

Sources: $\quad$ Fichier sectoriel RELIG

Fichier BALSAC. (Saguenay)

Les résultats saguenayens, appuyés sur des données très complètes et sur une mesure extrêmement précise du phénomène, ne supportent pas cet énoncé (tableaux 2, 3 et 4). Il n'y a pas d'écart entre la dimension des familles d'origine des prêtres et des pères, d'une part, et des témoins, d'autre part. Chez les religieuses et chez les frères, l'écart va dans le sens attendu, mais il est de quelques décimales seulement dans le premier cas et il est inférieur à l'unité dans le second, ce qui n'est guère probant. En ce qui concerne le Saguenay, il faut donc de toute évidence conclure ici à l'hypothèse nulle.

\section{C - La structure familiale}

Des nombreuses entrevues que nous avons réalisées dans cette région entre 1968 et 1985, il ressort que les parents trouvaient souvent leur intérêt à «placer» un fils ou une fille dans une communauté religieuse. En effet, avant la Seconde Guerre mondiale, la contraception était encore assez peu pratiquée et les familles dites complètes comptaient souvent de 8 à 10 enfants en moyenne ${ }^{21}$ et il arrivait assez souvent que les couples usent un peu de leur autorité pour susciter ou confirmer une vocation et diminuer ainsi la charge familiale. On peut s'attendre alors à ce que ce facteur ait pu jouer encore davantage au sein des familles brisées à la suite du décès prématuré d'un des conjoints. Encore une fois cependant, nos données démentent cet énoncé; l'écart observé entre le groupe cible et le groupe témoin va même dans le sens contraire (tableau 5), et ceci pour toutes les sous-périodes, sauf

21. G. Bouchard et R. Roy, «Fécondité et alphabétisation au Saguenay et au Québec (XIX ${ }^{\mathrm{e}}-\mathrm{XX}^{\mathrm{e}}$ siècles)», Annales de démographie historique (1991): 173-201. 
Tableau 4

Dimensions comparées (nombre moyen de naissances vivantes) des familles

d'origine des religieuses et des témoins, selon le groupe professionnel

Saguenay, 1842-1947

\begin{tabular}{|c|c|c|c|c|c|c|c|c|c|c|c|c|}
\hline \multirow{3}{*}{$\begin{array}{c}\text { Sous- } \\
\text { périodes }\end{array}$} & \multicolumn{4}{|c|}{ Cultivateurs } & \multicolumn{4}{|c|}{ Autres professions } & \multicolumn{4}{|c|}{ Toutes professions } \\
\hline & \multicolumn{2}{|c|}{ Religieuses } & \multicolumn{2}{|c|}{ Témoins } & \multicolumn{2}{|c|}{ Religieuses } & \multicolumn{2}{|c|}{ Témoins } & \multicolumn{2}{|c|}{ Religieuses } & \multicolumn{2}{|c|}{ Témoins } \\
\hline & $\begin{array}{c}\mathrm{N} . \mathrm{de} \\
\text { familles }\end{array}$ & $\begin{array}{l}\text { N. moyen } \\
\text { d'enfants }\end{array}$ & $\begin{array}{c}\mathrm{N} . \mathrm{de} \\
\text { familles }\end{array}$ & $\begin{array}{l}\text { N. moyen } \\
\text { d'enfants }\end{array}$ & $\begin{array}{c}N . \text { de } \\
\text { familles }\end{array}$ & $\begin{array}{l}\text { N. moyen } \\
\text { d'enfants }\end{array}$ & $\begin{array}{c}\text { N. de } \\
\text { familles }\end{array}$ & $\begin{array}{r}\text { N. moyen } \\
\text { d'enfants }\end{array}$ & $\begin{array}{c}\mathrm{N} . \mathrm{de} \\
\text { familles }\end{array}$ & $\begin{array}{l}\text { N. moyen } \\
\text { d'enfants }\end{array}$ & $\begin{array}{c}\text { N. de } \\
\text { familles }\end{array}$ & $\begin{array}{l}\text { N. moyen } \\
\text { d'enfants }\end{array}$ \\
\hline $1882-1899$ & 31 & 11,7 & 745 & 10,9 & 6 & 10,0 & 200 & 9,7 & 37 & 10,9 & 945 & 10,3 \\
\hline $1900-1947$ & 329 & 11,2 & 3818 & 10,9 & 204 & 10,9 & 2988 & 10,0 & 533 & 11,1 & 6806 & 10,5 \\
\hline Ensemble & 360 & 11,3 & 4563 & 10,9 & 210 & 10,8 & 3188 & 10,0 & 570 & 11,1 & 7751 & 10,5 \\
\hline
\end{tabular}

Sources: $\quad$ Fichier sectoriel RELIG

Fichier BALSAC (Saguenay) 
Tableau 5

Proportion de familles brisées chez le personnel religieux

et chez les témoins

Saguenay, 1882-1947 (a)

\begin{tabular}{||c|c|c|c|c|c|c|c|c||}
\hline \multirow{2}{*}{$\begin{array}{c}\text { Sous- } \\
\text { périodes }\end{array}$} & \multicolumn{2}{|c|}{ Personnel masculin } & \multicolumn{2}{c|}{$\begin{array}{c}\text { Personnel féminin } \\
\text { (religieuses) }\end{array}$} & \multicolumn{2}{c|}{$\begin{array}{c}\text { Ensemble du personnel } \\
\text { religieux }\end{array}$} & \multicolumn{2}{c|}{$\begin{array}{c}\text { Familles d'origine } \\
\text { des témoins }\end{array}$} \\
\cline { 2 - 9 } & $\begin{array}{c}\text { N. total de } \\
\text { familles }\end{array}$ & $\begin{array}{c}\text { \% de familles } \\
\text { briśes }\end{array}$ & $\begin{array}{c}\text { N. total de } \\
\text { familles }\end{array}$ & $\begin{array}{c}\text { \% de familles } \\
\text { brisées }\end{array}$ & $\begin{array}{c}\text { N. total de } \\
\text { familles }\end{array}$ & $\begin{array}{c}\% \text { de familles } \\
\text { briśes }\end{array}$ & $\begin{array}{c}\text { N. total de } \\
\text { familles }\end{array}$ & $\begin{array}{c}\% \text { de familles } \\
\text { briśes }\end{array}$ \\
\hline $1882-1891$ & 10 & 10,0 & 11 & 0,0 & 21 & 4,8 & 1157 & 7,0 \\
$1892-1901$ & 13 & 0,0 & 30 & 6,7 & 43 & 4,7 & 1366 & 7,2 \\
$1902-1911$ & 16 & 0,0 & 48 & 4,2 & 64 & 3,1 & 1720 & 8,4 \\
$1912-1921$ & 33 & 9,1 & 86 & 7,0 & 119 & 7,6 & 2547 & 9,7 \\
$1922-1931$ & 59 & 5,1 & 168 & 8,9 & 227 & 7,9 & 2970 & 10,1 \\
$1932-1941$ & 94 & 10,6 & 199 & 12,6 & 293 & 11,9 & 4352 & 11,6 \\
$1942-1947$ & 48 & 2,1 & 71 & 1,4 & 119 & 1,7 & 3024 & 9,9 \\
\hline $1882-1947$ & 273 & 6,6 & 613 & 8,3 & 886 & 7,8 & 17136 & 9,8 \\
\hline
\end{tabular}

(a) Les familles brisées prématurément sont celles où l'un ou les deux conjoints décède(nt) moins de 10 ans après le mariage (avant 32 ans pour les femmes, avant 36 ans pour les hommes). Dans le groupe cible comme dans le groupe témoin, près de $20 \%$ des familles ont dú être exclues parce qu'elles ne pouvaient être observées assez longtemps, à cause d'une émigration.

\section{Sources: $\quad$ Fichier sectoriel RELIG}


1932-1941. On notera aussi que, dans l'ensemble, la proportion de familles brisées demeure plutôt basse.

Ces résultats invitent à relativiser le témoignage des données orales et à utiliser celles-ci avec prudence. Les ruptures familiales suivies de remariages ont certes favorisé l'entrée en religion dans certains cas, mais il s'agit d'un phénomène très minoritaire dans l'ensemble des modalités de recrutement. Contrairement aussi à une affirmation souvent entendue, il n'est pas évident que le phénomène ait surtout affecté les filles ${ }^{22}$.

\section{D - La mobilité géographique et professionnelle}

Dans la mesure où les changements de résidence et de profession peuvent être associés à une instabilité préjudiciable à la cohésion familiale et à l'encadrement des enfants, on soupçonne qu'ils aient pu exercer une influence négative sur le recrutement religieux. Ce n'est pas le cas. Les familles d'origine du personnel religieux et des témoins ont vécu le même nombre de déplacements et de changements d'emploi (tableaux non reproduits). La mobilité n'était donc pas une source de perturbation.

En ce qui concerne la mobilité géographique, il est à noter aussi que, lorsqu'un fils ou une fille entrait en religion dans une communauté hors Saguenay, une fois sur quatre seulement la famille quittait elle aussi le Saguenay. Ce phénomène était sensiblement plus rare lorsque l'enfant (fils ou fille) demeurait au Saguenay.

\section{E - Le rang de naissance}

Selon une représentation encore une fois assez largement accréditée, ce sont surtout les plus jeunes enfants qui étaient susceptibles d'être poussés vers la vie religieuse; le couple avait alors acquis un peu d'aisance et pouvaient s'offrir ce privilège qui procurait en même temps une assurance pour le ciel. Sauf exception, les données saguenayennes contredisent aussi ce modèle. Comme on peut le constater au tableau 6, aucune relation nette n'associait rang de naissance et entrée en religion ${ }^{23}$. Un écart semble ressortir chez les pères en faveur du dernier quartile; mais dans ce cas, il faut se méfier des petits nombres. Un deuxième calcul, analogue, a été effectué mais en le limitant cette fois au rang de naissance des fils (pour les prêtres, pères, frères) ou

22. Référence aux cas de remariage d'un veuf donnant lieu à des conflits entre ses filles et leur belle-mère.

23. L'analyse a été conduite à l'échelle des quartiles plutôt qu'à celle des rangs de naissance afin d'éviter les trop petits effectifs. 
Tableau 6

\section{Répartition des membres du personnel religieux selon leur rang (quartile) de naissance \\ Saguenay, 1882-1947}

\begin{tabular}{|c|c|c|c|c|c|c|c|c|c|c|}
\hline \multirow[t]{2}{*}{$\begin{array}{c}\text { Rang de } \\
\text { naissance }\end{array}$} & \multicolumn{2}{|c|}{ Prêtres } & \multicolumn{2}{|c|}{ Pères } & \multicolumn{2}{|c|}{ Frères } & \multicolumn{2}{|c|}{$\begin{array}{l}\text { Ensemble du } \\
\text { personnel } \\
\text { masculin }\end{array}$} & \multicolumn{2}{|c|}{ Religieuses } \\
\hline & Nombre & $\%$ & Nombre & $\%$ & Nombre & $\%$ & Nombre & $\%$ & Nombre & $\%$ \\
\hline $1^{\text {er }}$ quartile & 38 & 29,2 & 8 & 17,8 & 16 & 16,6 & 62 & 22,9 & 177 & 25,5 \\
\hline $2^{\circ}$ quartile & 20 & 15,4 & 9 & 20,0 & 28 & 29,2 & 57 & 21,0 & 190 & 27,4 \\
\hline $3^{c}$ quartile & 37 & 28,5 & 8 & 17,8 & 28 & 29,2 & 73 & 26,9 & 192 & 27,7 \\
\hline $4^{\circ}$ quartile & 35 & 26,9 & 20 & 44,4 & 24 & 25,0 & 79 & 29,2 & 135 & 19,4 \\
\hline Total & 130 & 100,0 & 45 & 100,0 & 96 & 100,0 & 271 & 100,0 & 694 & 100,0 \\
\hline
\end{tabular}

Notes: Pour les fins du calcul, seules les familles comptant au moins 4 enfants ayant survécu jusqu'à l'âge adulte ont été retenues. Les familles au sein desquelles les rangs de naissance ne pouvaient être établis avec certitude ont été écartées.

Pour le regroupement en quartiles, les chiffres impairs ont été arrondis au plus près; en cas d'égalité, le hasard a tranché.

Sources: $\quad$ Fichier sectoriel RELIG

Fichier BALSAC (Saguenay) 
Tableau 7

Répartition des membres du personnel religieux selon leur rang (aíné, cadet) de naissance Saguenay, 1882-1947

\begin{tabular}{|c|c|c|c|c|c|c|c|c|c|c|}
\hline \multirow[t]{2}{*}{$\begin{array}{l}\text { Rang } \\
\text { (statut) }\end{array}$} & \multicolumn{2}{|c|}{ Prêtres } & \multicolumn{2}{|c|}{ Pères } & \multicolumn{2}{|c|}{ Frères } & \multicolumn{2}{|c|}{$\begin{array}{c}\text { Ensemble du } \\
\text { personnel } \\
\text { masculin }\end{array}$} & \multicolumn{2}{|c|}{ Religieuses } \\
\hline & Nombre & $\%$ & Nombre & $\%$ & Nombre & $\%$ & Nombre & $\%$ & Nombre & $\%$ \\
\hline Cadet & 24 & 16,7 & 10 & 21,3 & 8 & 8,2 & 42 & 14,5 & 61 & 8,5 \\
\hline Aîné & 34 & 23,6 & 7 & 14,9 & 9 & 9,2 & 50 & 17,3 & 110 & 15,4 \\
\hline Autres rangs & 86 & 59,7 & 30 & 63,8 & 81 & 82,6 & 197 & 68,2 & 545 & 76,1 \\
\hline TOTAL & 144 & 100,0 & 47 & 100,0 & 98 & 100,0 & 289 & 100,0 & 716 & 100,0 \\
\hline
\end{tabular}

Note: Les familles au sein desquelles les rangs de naissance ne pouvaient être établis avec certitude ont été écartées.

Sources: $\quad$ Fichier sectoriel RELIG

Fichier BALSAC (Saguenay) 
des filles (pour les religieuses). Il a donné des résultats similaires (données non reproduites).

Un troisième test avait pour but d'isoler les statuts d'aînés et de cadets (tableau 7). Mais là non plus, il n'a pas été possible de dégager un quelconque modèle préférentiel même en restreignant les calculs aux enfants de même sexe (données non reproduites). Enfin, tous les tests qui précèdent ont aussi été réalisés en faisant intervenir la variable professionnelle. Les résultats demeurent inchangés.

Toutes ces conclusions confirment une série d'analyses réalisées dans le cadre d'un autre projet de l'IREP concernant la reproduction familiale au sein de la paysannerie saguenayenne. À l'aide de divers indicateurs (choix de carrière des enfants, choix du donataire du patrimoine, niveaux d'alphabétisation...), il s'est avéré impossible de déceler des pratiques préférentielles ou des clivages significatifs reliés au rang de naissance ${ }^{24}$. Nous avons imputé ce phénomène au fait d'une collectivité neuve, encore faiblement hiérarchisée, où un certain égalitarisme est servi par la flexibilité inhérente au contexte de peuplement.

\section{2 - L'APPARENTEMENT}

À propos de l'univers familial comme matrice du recrutement religieux, nous avons cru important de bien distinguer quatre facteurs: la taille de la famille, le bassin familial de recrutement (modèle des «pépinières» familiales?), l'influence de la parentèle et un possible effet intergénérationnel - ou transmission familiale (nous dirons à l'avenir: héritabilité). Le premier facteur a été abordé plus haut. La présente partie est consacrée aux trois autres.

\section{A - Le bassin de recrutement}

La première hypothèse à valider est celle voulant qu'une minorité de familles ait fourni à l'Église la majeure partie de son personnel. En ce qui concerne le Saguenay comme de nombreuses autres populations sans doute, diverses études livrent des exemples spectaculaires de familles d'où sont issus trois, quatre ou cinq prêtres, religieux ou religieuses. Comme on s'en doute, les annales saguenayennes recèlent de beaux cas de ce genre: ici, toutes les filles $(\mathrm{N}=5)$ d'une famille de 12 enfants deviennent religieuses; là, quatre garçons sur cinq accèdent à la prêtrise ${ }^{25}$, etc. Mais comme on peut le penser aussi, ces cas

24. Gérard Bouchard, «Rang de naissance et destin socio-économique dans la région du Saguenay, Québec (XIX $\mathrm{XX}^{\mathrm{e}}-\mathrm{XX}^{\mathrm{c}}$ s.)», Martine Segalen, Georges Ravis-Giordani, dirs., Les Cadets (Paris, CNRS Éditions, 1994), 95-108.

25. Archives nationales du Québec à Chicoutimi, Mémoires d'Anciens no 560 (57-58). 
relèvent très souvent du domaine de l'anecdote et ont finalement peu à voir avec la réalité d'ensemble.

Tout d'abord, il est vrai que, quelle que soit la sous-période considérée, une minorité de familles seulement alimentait les rangs de l'Église saguenayenne. Dans chaque cohorte décennale entre 1882 et 1947 , la proportion des familles qui ne fournissaient aucune vocation a oscillé entre $89 \%$ et $96 \%$. En lui-même toutefois, ce résultat n'est nullement significatif puisque les gens d'Église constituaient une petite élite ${ }^{26}$. Très minoritaires, ils ne pouvaient provenir par définition que d'une minorité de familles. C'est plutôt le mode de recrutement au sein de ce groupe de familles qui est déterminant. Or, sur l'ensemble de la période, le nombre de ces familles qui ont fourni la totalité de la population des clercs, religieux et religieuses $(\mathrm{N}=1552)$ s'élève à 1 195. De ce nombre, 935 (soit près des quatre cinquièmes) n'ont donné qu'un membre au personnel religieux (tableau 8). À l'autre bout de la distribution, les familles ayant fourni trois vocations ou plus ne représentent que $5,1 \%$ des familles ayant contribué (et $0,5 \%$ des familles du Saguenay). Par ailleurs, les vocations issues de ces familles ne comptent que pour 14,2\% de l'ensemble des vocations.

Ces données doivent être interprétées avec nuance, surtout en l'absence de repères comparatifs. Mais compte tenu du fait que le personnel religieux constitue une minorité qui ne peut être issue que d'un nombre restreint de familles, on est plutôt frappé par la dispersion du recrutement familial, la très grande majorité des familles concernées ne donnant qu'un seul membre à l'Église. On est donc assez loin du modèle de la «pépinière». Ajoutons que cet énoncé vaut pour tous les groupes socioprofessionnels (données non reproduites). Enfin, les familles ayant donné deux vocations ou plus ne se distinguent des autres ni sous le rapport de l'habitat (rural/urbain) ni sous le rapport de la mobilité géographique ou professionnelle. De plus, les familles ayant fourni de 2 à 5 vocations sont à peine plus grosses que les autres (11,8 enfants contre 10,5).

\section{$B$ - Le recrutement dans la parentèle}

La conclusion précédente est cependant compatible avec un mode de recrutement axé sur la famille dite étendue ou sur la parentèle. C'est effectivement ce qui s'est produit. Nous avons pu le voir en mesurant l'apparentement dans l'une des communautés religieuses actives dans la région (Augustines Hospitalières) et pour laquelle 186 membres ont été recensés entre 1882 et 1947. Un groupe témoin

26. G. Bouchard et R. Thibeault, loc. cit., tableaux 1 et 2 . 
Tableau 8

Nombre de vocations religieuses par famille Saguenay, 1882-1947

\begin{tabular}{|c|c|c|c|c||}
\hline \multirow{2}{*}{$\begin{array}{c}\text { Nombre de } \\
\text { vocations par } \\
\text { famille }\end{array}$} & \multicolumn{2}{|c|}{$\begin{array}{c}\text { Nombre de } \\
\text { familles }\end{array}$} & \multicolumn{2}{c|}{$\begin{array}{c}\text { Nombre total de } \\
\text { clercs, religieux, } \\
\text { religieuses }\end{array}$} \\
\cline { 2 - 5 } & Nombre & $\%$ & Nombre & $\%$ \\
\hline 1 & 935 & 78,2 & 935 & 60,2 \\
3 & 199 & 16,7 & 398 & 25,6 \\
4 & 36 & 3,0 & 108 & 7,0 \\
5 & 16 & 1,3 & 64 & 4,1 \\
6 & 7 & 0,6 & 35 & 2,3 \\
\hline Total & 195 & 100,0 & 1552 & 100,0 \\
\hline
\end{tabular}

Sources:

a été construit en appariant chaque religieuse avec une personne du même âge, du même sexe, de la même paroisse et issue du même milieu socioprofessionnel. Pour toutes les personnes du groupe cible et du groupe témoin, les ascendances $(\mathrm{N}=372)$ ont été construites intégralement sur trois générations (excluant celle du sujet ${ }^{27}$. Le coefficient de parenté (Phi), emprunté à la génétique des populations, a été calculé pour chacun des deux corpus ${ }^{28}$. La valeur du coefficient

27. Dans les deux groupes, plus de $95 \%$ des ancêtres ont pu être identifiés, incluant une bonne partie de ceux - assez nombreux - qui s'étaient mariés hors du Québec.

28. Ce coefficient fournit une évaluation globale de l'apparentement entre les sujets, à une profondeur généalogique donnée - ici: 3 générations, excluant le sujet. 
Tableau 9

Distribution des degrés de parenté observés entre chaque paire de sujets

Fichier des religieuses et fichier des témoins

\begin{tabular}{||c|c|c|c||}
\hline $\begin{array}{c}\text { Valeurs du } \\
\text { coefficient Phi } \\
(10 \times-4)\end{array}$ & $\begin{array}{c}\text { Degré de parenté } \\
\text { équivalent } \\
\text { (selon le droit } \\
\text { canonique) }\end{array}$ & $\begin{array}{c}\text { Nombre de } \\
\text { paires } \\
\text { de religieuses }\end{array}$ & $\begin{array}{c}\text { Nombre de } \\
\text { paires } \\
\text { de témoins }\end{array}$ \\
\hline 2500 & $1: 1$ & 32 & 1 \\
1250 & $1: 2$ & 23 & 17 \\
625 & $2: 3$ & 54 & 36 \\
312 & $3: 3$ & 82 & 65 \\
78 & $3: 4$ & 46 & 6 \\
39 & $>4$ & 3 & 1 \\
\hline
\end{tabular}

Note: Une même personne peut être apparentée avec une autre de plus d'une façon.

Sources: $\quad$ Fichier sectoriel RELIG

Fichier BALSAC (Saguenay)

s'avère effectivement plus élevée dans le groupe cible que dans le groupe témoin $\left(9,15 \times 10^{-4}\right.$ contre $\left.2,4 \times 10^{-4}\right)$, bien que, en ce qui concerne le groupe cible, ce degré d'apparentement ne peut en aucune façon être considéré comme spectaculaire. Le détail de la comparaison est donné aux tableaux 9 et 10 . 
Distribution des sujets selon le nombre

de personnes apparentées dans le groupe cible des religieuses et dans le groupe témoin

\begin{tabular}{|c|c|c|c|c|}
\hline \multirow{2}{*}{$\begin{array}{l}\text { Nombre de personnes } \\
\text { apparentées }\end{array}$} & \multicolumn{2}{|c|}{ Religieuses } & \multicolumn{2}{|c|}{ Témoins } \\
\hline & Nombre & $\%$ & Nombre & $\%$ \\
\hline 0 & 51 & 27,4 & 62 & 33,3 \\
\hline 1 & 54 & 29,0 & 49 & 26,3 \\
\hline 2 & 15 & 8,0 & 29 & 15,6 \\
\hline 3 & 18 & 9,7 & 20 & 10,8 \\
\hline 4 & 14 & 7,5 & 12 & 6,5 \\
\hline 5 & 7 & 3,8 & 4 & 2,2 \\
\hline 6 & 2 & 1,1 & 3 & 1,6 \\
\hline 7 & 7 & 3,8 & 4 & 2,2 \\
\hline 8 & 2 & 1,1 & 1 & 0,5 \\
\hline 9 & 0 & - & 1 & 0,5 \\
\hline 10 & 3 & 1,6 & 1 & 0,5 \\
\hline 11 & 0 & - & 0 & 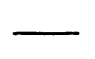 \\
\hline 12 & 5 & 2,7 & 0 & $\longrightarrow$ \\
\hline 13 & 1 & 0,5 & 0 & 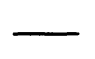 \\
\hline 14 & 0 & $\longrightarrow$ & 0 & 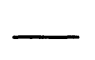 \\
\hline 15 & 2 & 1,1 & 0 & 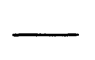 \\
\hline 16 & 4 & 2,2 & 0 & 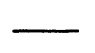 \\
\hline 17 & 0 & - & 0 & 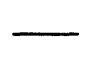 \\
\hline 18 & 0 & $\longrightarrow$ & 0 & - \\
\hline 19 & 1 & 0,5 & 0 & $\longrightarrow$ \\
\hline 20 & 0 & - & 0 & - \\
\hline Nombre Total & 186 & 100,0 & 186 & 100,0 \\
\hline $\begin{array}{l}\text { Moyenne } \\
\text { pondérée }\end{array}$ & - & 2,81 & - & 1,66 \\
\hline
\end{tabular}


Le tableau 9 montre bien que, pour tous les degrés de parenté, les religieuses supplantent les témoins. On constate aussi que plus la parenté est proche, plus les écarts sont considérables, ce qui révèle que le recrutement touchait de préférence le voisinage immédiat de la famille conjugale. Par conséquent, les religieuses se retrouvaient en terrain plus que familier dans leur communauté, ce qu'illustre le tableau 10. Comme on s'y attend, les écarts entre les deux groupes se creusent surtout aux valeurs élevées (10 et plus). Nous avons aussi représenté aux graphiques 1 et 2 les généalogies de deux cas un peu exceptionnels. Le premier cas implique 19 religieuses apparentées au sujet de départ (dont 12 cousines et petites cousines), le second 16.

Ce phénomène n'est pas rare. Au Saguenay, un sondage réalisé dans une autre communauté religieuse laisse entrevoir un phénomène analogue: sur 64 membres, 42 étaient apparentés. Dans son ouvrage sur les sœurs de la Miséricorde et de la congrégation Notre-Dame à Montréal, Marta Danylewycz ${ }^{29}$ fournit des indications allant dans le même sens. Toutes ces données attestent un mode de recrutement qui s'appuyait largement sur les réseaux de la parenté, mettant à contribution ses courroies de diffusion et ses solidarités.

\section{C - Un effet intergénérationnel}

Une dernière question porte sur ce que nous avons appelé l'héritabilité des vocations religieuses. Nous savons qu'à chaque génération une minorité de familles alimentait les rangs du personnel religieux. Mais, d'une génération à l'autre, le recrutement puisait-il dans ces mêmes familles? En d'autres mots, existait-il des lignées sacerdotales ou religieuses? Pour répondre à cette question, nous avons eu recours encore une fois au fichier RELIG et à la population de référence. Dans un premier temps, les vocations postérieures à $1914(\mathrm{~N}=619)$ ont été examinées une à une pour vérifier si dans leur ascendance immédiate - en l'occurrence les oncles et tantes paternels et maternels - il se trouvait déjà un membre du personnel religieux. Dans un deuxième temps, et pour les mêmes années (1915-1947), les 20382 témoins ont été passés en revue et soumis à la même interrogation. Les résultats sont présentés au tableau 11.

D'abord, il s'avère qu'il existe incontestablement un effet intergénérationnel. De fait, la proportion de sujets comptant un membre du personnel religieux à la génération antérieure est deux fois plus élevée dans le groupe cible que dans le groupe témoin. Cet écart paraît en

29. Marta Danylewycz, Taking the Veil: an Alternative to Marriage, Motherhood, and Spinsterhood in Quebec, 1840-1920 (Toronto, McClelland and Stewart, 1987), 112-113. 


\section{Graphique 1}

Apparentement d'une religieuse de la communauté des Augustines Hospitalières (Saguenay). Exemple 1

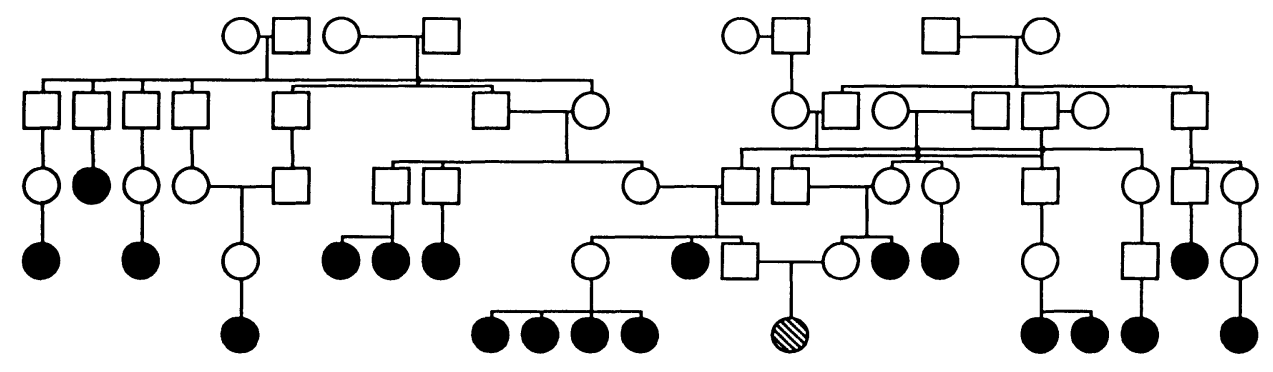

Sources: Fichier sectoriel RELIG Fichier BALSAC (Saguenay)

$$
\text { Légende: } \begin{aligned}
\mathbb{Q} & =\text { sujet de départ (Ego) } \\
& =\text { apparentées } \\
\bigcirc & =\text { femme } \\
\square & =\text { homme }
\end{aligned}
$$

\section{Graphique 2}

Apparentement d'une religieuse de la communauté des Augustines Hospitalières (Saguenay). Exemple 2

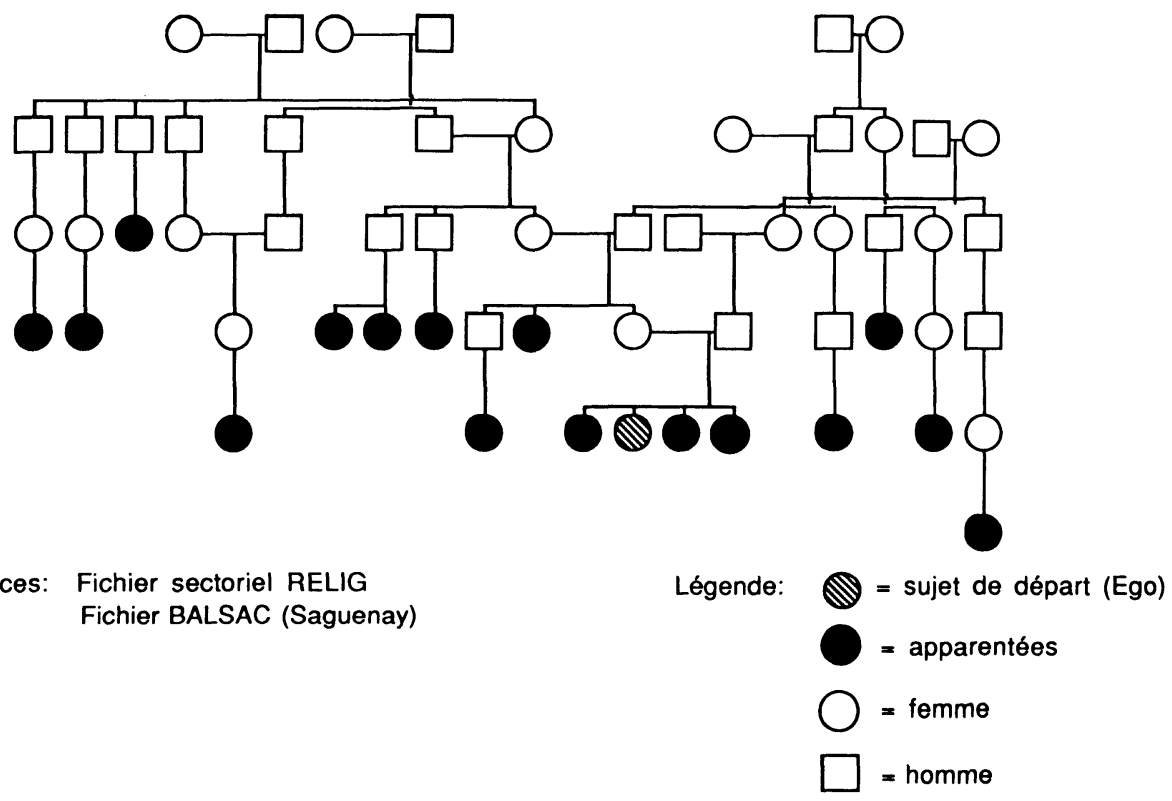


Proportion de clercs, religieux et religieuses ayant compté un membre du personnel religieux parmi leurs oncles et tantes paternels ou maternels

\begin{tabular}{|c|c|c|c|c|}
\hline \multirow[t]{2}{*}{ Sous-groupes } & \multicolumn{2}{|c|}{$\begin{array}{l}\text { Entré(e)s en religion } \\
\text { entre } 1915 \text { et } 1930\end{array}$} & \multicolumn{2}{|c|}{$\begin{array}{l}\text { Entré(e)s en religion } \\
\text { entre } 1931 \text { et } 1947\end{array}$} \\
\hline & $N$ (a) & $\%$ (b) & $\mathbf{N}$ & $\%$ \\
\hline Prêtres & 59 & 27,1 & 67 & 26,9 \\
\hline Pères & 9 & 11,1 & 45 & 24,4 \\
\hline Frères & 25 & 8,0 & 74 & 20,0 \\
\hline Religieuses & 271 & 23,2 & 69 & 23,2 \\
\hline $\begin{array}{l}\text { Ensemble du } \\
\text { personnel } \\
\text { religieux }\end{array}$ & 364 & 22,6 & 255 & 23,1 \\
\hline Témoins (c) & 6789 & 9,6 & 13593 & 12,0 \\
\hline
\end{tabular}

Notes: (a) Nombre de vocations religieuses durant la sous-période

(b) Proportion de sujets ayant un oncle ou une tante en religion

(c) Familles témoins composant la population de référence

Sources: $\quad$ Fichier sectoriel RELIG

Fichier BALSAC (Saguenay) 
outre relativement stable, indépendant des sous-périodes et des catégories $^{30}$. Cependant, les proportions relevées dans le groupe cible $(22,6 \%$ et $23,1 \%$ ) demeurent tout de même modestes, et on ne peut pas dire qu'elles confirment d'une manière péremptoire la thèse des lignées sacerdotales (telle que formulée notamment par Philippe Garigue ${ }^{31}$ ). En outre, des nuances importantes doivent être apportées. Ainsi, lorsqu'on ventile les données selon les groupes socioprofessionnels, on s'aperçoit que l'effet intergénérationnel ne se manifeste que dans les familles de travailleurs manuels (urbains ou ruraux) (tableau non reproduit).

\section{3 - CONCLUSION}

En définitive, il ressort des analyses qui précèdent que le recrutement du personnel religieux saguenayen était assez peu sensible à des variables comme la dimension et la structure de la famille, le rang de naissance, la mobilité géographique et socioprofessionnelle. Parmi les facteurs analysés ici, seule l'alphabétisation paraît avoir exercé une influence positive. Quant aux variables reliées à l'apparentement, on observe que le recrutement favorisait effectivement la parentèle et que certains éléments d'héritabilité apparaissaient, bien qu'à un degré modeste. Quant au modèle de la «pépinière», il ne se vérifie pas. On peut s'étonner de ne pas retrouver ici une emprise plus décisive des articulations du social; nous sommes tentés d'y voir le fait d'une population neuve, trop proche encore de ses racines et qui n'a pas encore pleinement déployé ses structures, ses hiérarchies, ses clivages.

Ces données font par ailleurs supposer que des facteurs culturels ont pu jouer un rôle prédominant. Nous l'avons déjà vu pour l'alphabétisation. Dans le même sens, il appert aussi - et sans surprise que la variabilité du sentiment religieux, ou de la sensibilité au sacré, rend sans doute compte d'une bonne partie de nos résultats. C'est ce que suggère un dernier test que nous avons effectué. Le délai qui s'écoulait entre la naissance et le baptême d'un enfant peut être utilisé comme un indicateur de l'intensité de la foi catholique; on sait que les couples croyants avaient très peur de voir leur nouveau-né décéder avant d'être baptisé, ce qui le vouait à jamais aux limbes tant redoutés dans la représentation populaire ${ }^{32}$. On s'empressait donc de le faire

30 À cause des petits effectifs, il ne faut sans doute pas trop porter attention aux pères et aux frè.res, pour la sous-période 1915-1930.

31 Philippe Garigue, «St. Justin, a Case-Study in Rural French Canadian Social Organization», Canadian Journal of Economics and Political Science (1956): 301-318.

32. On pouvait certes l'ondoyer au besoin mais, selon les anciens Saguenayens, rien ne pouvait remplacer la main sacrée du prêtre. 
Tableau 12

Délai moyen entre la naissance et le baptême dans les familles du personnel religieux et dans les familles témoins

Familles de cultivateurs seulement (Saguenay)

\begin{tabular}{||c|c|c|c|c|c|c||}
\hline \multirow{2}{*}{ Sous-période (b) } & \multicolumn{3}{|c|}{ Groupe cible } & \multicolumn{3}{c|}{ Groupe témoin } \\
\cline { 2 - 7 } & $\begin{array}{c}\text { Nombre de } \\
\text { naissances (a) }\end{array}$ & $\begin{array}{c}\text { Délai moyen } \\
\text { (jours) }\end{array}$ & $\begin{array}{c}\text { \% de baptêmes dans } \\
\text { un délai inférieur à } \\
48 \text { heures }\end{array}$ & $\begin{array}{c}\text { Nombre de } \\
\text { naissances (a) }\end{array}$ & $\begin{array}{c}\text { Délai moyen } \\
\text { jours) }\end{array}$ & $\begin{array}{c}\text { \% de baptêmes célébrés } \\
\text { le jour même ou le } \\
\text { lendemain de la naissance }\end{array}$ \\
\hline $1852-1871$ & 607 & 1,7 & 89,8 & 10463 & 2,4 & 89,4 \\
$1872-1891$ & 1340 & 1,0 & 93,8 & 16668 & 1,8 & 91,6 \\
$1892-1911$ & 2758 & 1,2 & 93,8 & 27373 & 1,7 & 94,0 \\
$1912-1931$ & 2188 & 0,8 & 96,2 & 24012 & 9,2 & 94,9 \\
\hline Total & 6893 & 1,1 & 94,2 & 78516 & 1,7 & 93,2 \\
\hline
\end{tabular}

Notes: (a) Toutes les naissances vivantes ont été prises en compte dans chaque famille.

(b) Les sous-périodes correspondent aux années de naissance des membres du personnel religieux, de leurs frères et sœurs ainsi que des témoins. 
baptiser très tôt après sa naissance - pratique qui, durant les tempêtes d'hiver, mettait paradoxalement en danger la vie de l'enfant. Jusqu'au milieu du $\mathrm{XX}^{\mathrm{e}}$ siècle, plus de quatre baptêmes sur cinq étaient ainsi célébrés moins de 48 heures après la naissance de l'enfant. Nous avons comparé ce délai (sous deux formes: délai moyen en jours et proportion des baptêmes célébrés le jour même ou le lendemain de la naissance) dans les familles du groupe cible (sujets entrés en religion de même que leurs frères et sœurs) et dans celles de la population de référence (tableau 12). Il est à noter que, dans les deux cas, le groupe cible se démarque dans le sens attendu, attestant un sentiment religieux plus éveillée ${ }^{33}$. Signalons en outre que les écarts s'accentuent toujours dans le même sens - lorsqu'on isole, au sein du groupe cible, les familles qui ont fourni plus d'un sujet à l'Église.

La croyance et la dévotion ne suivaient donc pas des chemins aisément prévisibles selon les indicateurs sociologiques habituels. Mais cette conclusion est sujette à révision. Il reste en effet à évaluer l'influence des variables économiques, surtout la richesse des familles, sur le recrutement religieux ${ }^{34}$. D'autres facteurs de même nature ont pu jouer aussi, par exemple la saturation des espaces agraires ${ }^{35}$. Par contre, il ne semble pas que la dot ait été un facteur important de sélection socio-économique. Le montant en était certes relativement élevé (par exemple: de $300 \$$ à $500 \$$ chez les Augustines Hospitalières à la fin du XIX ${ }^{\mathrm{c}}$ siècle, alors qu'un journalier gagnait environ $1 \$$ par jour), mais peu de familles la payaient. Selon un relevé effectué par Normand Perron ${ }^{36}$ dans cette même communauté, près de la moitié des postulantes ont été admises gratuitement entre 1884 et 1950. Les données orales que nous avons nous-mêmes recueillies à ce sujet confirment ce résultat et donnent à croire que la dot n'était presque jamais réglée complètement ${ }^{37}$.

Il reste à intégrer ces résultats empiriques dans une perspective plus large d'analyse des élites dans la région du Saguenay. Il va de soi que les investissements méthodologiques exigés par la construction et

33. La contraction du délai avec le temps est imputable sans doute à l'amélioration des moyens de transport.

34. C'est ce que nous faisons dans le cadre d'un autre projet de recherche sur les inégalités et les structures sociales au Saguenay.

35.. M. Danylewycz, op. cit., 52-54.

36 N. Perron, op. cit., 192.

37. Il s'agit peut-être d'une pratique très ancienne au Canada français. Dans son étude sur les religieuses entre 1639 et 1800, Micheline D'Allaire établit que, malgré le montant élevé, la dot n'a pas empêché un recrutement croissant de «petites gens». Micheline D'Allaire, Les dots des religieuses au Canada français, 1639-1800. Étude économique et sociale (Montréal, Hurtubise HMH, coll. «Cahiers du Québec, Histoire», n 86, 1986), 244 p. 
la confrontation des diverses variables ralentissent la démarche. Mais on s'accordera aisément sur leur caractère indispensable, si l'on en juge par les stéréotypes qu'ils permettent de récuser. 